Revue d'histoire de l'Amérique française

REVUE D.HISTOIRE DE L'AMÉRIQUE FRANÇAISE

\title{
Une société à hauteur d'homme : La Nouvelle-France
}

\section{Guy Frégault}

Volume 17, numéro 1, juin 1963

URI : https://id.erudit.org/iderudit/302249ar

DOI : https://doi.org/10.7202/302249ar

Aller au sommaire du numéro

Éditeur(s)

Institut d'histoire de l'Amérique française

ISSN

0035-2357 (imprimé)

1492-1383 (numérique)

Découvrir la revue

Citer cet article

Frégault, G. (1963). Une société à hauteur d'homme : La Nouvelle-France. Revue d'histoire de l'Amérique française, 17(1), 3-11. https://doi.org/10.7202/302249ar d'utilisation que vous pouvez consulter en ligne.

https://apropos.erudit.org/fr/usagers/politique-dutilisation/ 


\section{UNE SOCIÉTÉ À HAUTEUR D'HOMME : LA NOUVELLE-FRANCE *}

Lorsque j'étais un historien professionnel, il arrivait que l'on me priât de tenir des propos sur des sujets étrangers aux questions qui s'inscrivaient dans le cercle de mes préoccupations habituelles. Maintenant que je suis devenu un modeste amateur d'histoire, il se trouve que des historiens de profession, réunis en journée d'études, m'invitent à leur adresser la parole dans des circonstances telles que je ne vois pas comment je pourrais m'abstenir de leur parler d'histoire. J'ai d'abord pensé à user d'un subterfuge qui doit être permis, puisqu'il est impuni, et à me réfugier dans le décor somptueux de la théorie historique: n'est-il pas de bonne tradition que les amateurs proposent aux professionnels des considérations méthodologiques et les exhortent admirablement à bien pratiquer leur métier ? Vous vous souvenez tous de ce personnage de Stephen Leacock qui enseigna la natation avec un succès prodigieux jusqu'au jour où, étant tombé dans la piscine, il apparut qu'il ne savait pas nager. Je n'ai pas pu me résigner à un succès aussi certain. Et, devenant l'élève du professeur que j'ai été - c'est-à-dire me rappelant tant bien que mal ce que j'ai déjà enseigné j'ai choisi d'évoquer le Canada sous le régime français.

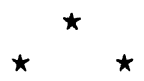

C'était l'époque où notre pays était à la fois normal et intéressant. Normal, parce qu'on y trouvait, comme partout, des puissants et des faibles, des riches et des pauvres, des citadins et des ruraux, des lettrés et des ignorants, des têtes

* Allocution prononcée à l'occasion du Congrès de l'Institut d'Histoire de l'Amérique française, le 27 avril 1963. 
médiocres et des esprits brillants, des personnes qui pratiquaient la vertu et d'autres qui ne s'y adonnaient guère qu'à l'extrémité. En somme, une société à hauteur d'homme. Intéressant, il fallait bien que le Canada le fût pour retenir comme il le faisait l'attention de ceux qui, après être entrés en contact avec lui, ont laissé ces relations, ces mémoires, ces chroniques et ces récits à travers lesquels il nous est possible aujourd'hui de ressaisir la vie que menaient ses habitants vers le milieu du 18e siècle.

Essayons de nous mettre à la place d'un de ces voyageurs. Parce qu'il n'a pas eu l'avantage de lire nos livres du 19e siècle, il ne s'étonne pas que son navire le dépose dans une ville. D'autre part, comme, à l'égal de ses contemporains, les chiffres le séduisent peu (contrairement à nous, qui en sommes fascinés), il ne cherchera jamais à savoir combien Québec compte d'habitants: Charlevoix, bon observateur pourtant, donne sans s'émouvoir à la capitale de la Nouvelle-France une population deux fois plus forte que celle qui y vit au moment où il $\mathrm{y}$ séjourne.

Puisque notre voyageur anonyme ne connaît rien à ce qu'écriront nos historiens, il n'éprouve aucune surprise à constater que la collectivité canadienne comporte, comme toutes les autres, des classes diverses dont les membres se reconnaissent à leur attitude, à leur prestige, à leurs préoccupations, à leurs privilèges, à leur niveau de vie et à leur conversation. A mille signes, il lui est aisé de voir qu'il est en terre française et en milieu colonial. Autour de lui, on s'exprime en français. "Ils parlent un français épuré, note Franquet, n'ont pas le moindre accent.» «Ils parlent avec aisance, leur accent est aussi bon qu'à Paris », renchérit Bougainville, qui leur reproche cependant d'user à tort et à travers de termes de marine; du reste, un ou deux compagnons d'armes de Montcalm s'excuseront auprès de leurs correspondants d'écrire moins bien qu'ils ne le faisaient en France, leur contact prolongé avec la société canadienne ayant enlevé quelque élégance à leur style.

C'est que colonie est à peu près synonyme de province. Lorsque Montcalm décrit Québec, le croquis qu'il en fait est celui d'une bonne agglomération provinciale. Trente ans plus tôt, 
Charlevoix avait donné, de la capitale du Canada, une impression analogue et d'une précision encore plus saisissante. Evoquant les conversations que tenait l'aristocratie locale, il avait remarqué: «Les nouvelles courantes se réduisent à bien peu de choses parce que le pays n'en fournit presque point et que celles de l'Europe arrivent tout à la fois, mais elles occupent une bonne partie de l'année.» Ce pays, cette époque qui ne fournissent presque point de nouvelles, des générations d'érudits et d'historiens les ont étudiés avec diligence et à peine ont-ils effleuré la masse de faits de toute sorte qui s'y sont bousculés. En somme, les fins causeurs de l'élite québecoise ont une attitude vraiment provinciale, authentiquement coloniale: ils dédaignent les choses du pays, ils ont le sentiment qu'il ne s'y passe rien et, pour prendre toute son envergure, leur esprit se donne l'air d'avoir besoin des vastes horizons de la conjoncture européenne.

Ce que signale Charlevoix doit être exact, parce que le goût des Canadiens pour les réalités extérieures se devine à la composition de leurs bibliothèques, où l'on trouve un peu de tout: des classiques latins et français, des écrivains contemporains - Montesquieu, Voltaire ... - des ouvrages de droit et de religion, de l'histoire: histoires de France, du Japon, de l'Hindoustan, de la Chine, de la Turquie, du Mexique, mais très peu de livres se rapportant au Canada. Les Relations des Jésuites étaient, semble-t-il, absentes de la plupart des bibliothèques. Benjamin Sulte a même voulu y voir une preuve de l'impopularité de ces religieux. La vérité toute simple est que les Canadiens ne se préoccupaient guère de ce qui s'écrivait sur leur patrie. En revanche, il leur arrivait non seulement de recevoir les gazettes européennes, mais de les faire relier pour les relire à loisir.

La société canadienne des années 1750 présente donc les traits caractéristiques d'une société coloniale. Elle évolue dans le cadre de l'empire français, qui lui fournit des hommes, de l'argent, du matériel, et aussi des idées, des techniques, une culture, en somme, et, en même temps, une direction, toutes choses dont une colonie ne peut pas se passer. Sa politique y trouve l'appui indispensable à toute collectivité en formation. 
On estime souvent que l'activité politique des Canadiens ne pouvait être que très réduite, vers 1750 , parce que, croit-on, toutes les décisions se prenaient à Versailles et que le gouvernement colonial n'avait d'autre fonction que de les appliquer. Mais les décisions de Versailles, qui les préparait, qui les inspirait ? Il n'est que d'avoir parcouru la correspondance échangée entre Québec et la Cour pour savoir que c'était, en général, le gouvernement canadien. Au surplus, il ne faudrait pas s'imaginer que la France traitât sa colonie en pays conquis. Le Canada ne faisait pas partie d'un empire étranger. Métropole et colonie étaient deux éléments homogènes d'un même monde français. Entre elles, il y avait non seulement communauté de culture, mais communauté de vie.

Si le gouverneur général et l'intendant représentaient le gouvernement métropolitain au Canada, ils étaient aussi les interprètes du Canada auprès de la mère-patrie. Concrètement, ils ne pouvaient pas gouverner seuls. Il leur fallait le concours d'un entourage formé d'officiers, de magistrats, de fonctionnaires, d'hommes d'affaires et de chefs spirituels. Comme partout ailleurs en Amérique, une classe dirigeante s'était formée et, comme partout ailleurs dans le monde, elle n'était pas dirigeante seulement de nom. Ainsi qu'il est normal de ce côté-ci du ciel, ce groupe comportait plus d'une équipe. Or, de telles équipes se renouvellent parce qu'elles se combattent et se déplacent. Il existe des «cabales », comme on dit à l'époque, des partis, dirait-on aujourd'hui. Et que sont les partis ? Des pyramides d'intérêts, sans aucun doute, en même temps que des cadres dont l'armature repose sur une certaine conception de l'intérêt public. Mais la politique a des hauts et des bas. Précisément, vers 1750 , on voit Bigot constituer son parti, comme Talon avait organisé le sien trois générations auparavant, et ce parti se compose de Canadiens et de Français; le plus grand profiteur du régime, Joseph Cadet, est né au Canada. L'autre parti, celui que déloge le groupe de l'intendant, commence à dénoncer l'adversaire, comme ses propres adversaires l'avaient dénoncé à l'époque agitée de Dupuy. Oui, la politique canadienne est beaucoup plus compliquée et infiniment moins désincarnée qu'on ne croirait à première vue. 
Le fait qu'il s'agisse d'une politique coloniale ajoute encore à sa complexité. Bien que les intérêts supérieurs de la colonie et ceux de la métropole se confondent, il est normal que les intérêts immédiats - même légitimes - des métropolitains et des coloniaux entrent en conflit. Ceux-ci ont leurs aspirations propres et, disons le mot, leurs propres ambitions. Vaudreuil représente bien les unes et les autres. Montcalm le fait passer pour francophobe. Rien de plus faux. En réalité, Montcalm incarne une forme déjà arriérée d'impérialisme, alors que l'attitude de Vaudreuil traduit un impérialisme plus évolué. Dans le cadre indispensable de l'empire, le gouverneur vise à renforcer les structures sociales et l'armature politique de la colonie, ce qui est à la fois d'un agent lucide de la métropole et d'un bon Canadien.

Ce sentiment de la patrie, Vaudreuil n'en a pas le monopole. Il le partage avec les classes dirigeantes du pays. Les trois groupes sociaux qui l'expriment avec le plus de précision sont les officiers, les commerçants et le clergé.

Les officiers appartiennent pour la plupart aux grandes familles seigneuriales qui se sont élevées au cours de la période dominée par la Compagnie des Habitants, puis au moment où Talon attachait à la colonie le régiment de Carignan. Un puissant esprit de corps les anime; il tient en partie à leur orgueil de caste et en partie à la nécessité où ils sont de défendre leurs positions contre des concurrents français.

Les marchands sont la seule classe qui se soit pourvue de son organisation propre. Ayant établi une bourse à Québec et à Montréal, ils élisent des syndics pour les représenter auprès des pouvoirs publics, et, à l'occasion, ces délégués parlent au nom de la population entière d'une ville.

Quoiqu'il se recrute un peu à tous les niveaux de la société, le clergé prend le ton que lui donnent ceux de ses membres issus des classes supérieures. Ces ecclésiastiques briguent naturellement les dignités, ils ne font pas la vie trop douce aux Français qui obtiennent parfois un rang élevé, et les évêques parlent souvent de leur humeur indépendante. 
Ainsi, les mêmes réactions et la même solidarité s'expriment dans les milieux les plus éveillés et les plus justement considérés de la société. L'attitude des notables semble bien traduire l'état d'esprit de la population qu'ils encadrent. Comment se comporte cette population ? J'aimerais avoir le temps de décrire les conditions dans lesquelles s'écoule l'existence des ouvriers des villes. Ce sont les grands oubliés de l'histoire de la NouvelleFrance. Mais, précisément parce qu'on les a négligés, les historiens qui s'adonneront à des études sociales n'auront rien à défaire et presque rien à corriger lorsqu'ils s'appliqueront à l'examen de leur genre de vie.

Si de la ville, on passe à la campagne, la situation qui se présente à l'observateur paraît, pour des raisons contraires, fort difficile à définir. Un fait s'impose cependant à l'attention: plusieurs témoignages donnent aux agriculteurs un haut niveau de vie. Lahontan, au début du siècle, et Montcalm, cinquante ans plus tard, comparent les ruraux à des « petits gentilhommes de France ». Hocquart écrit qu'ils «n'ont point l'air grossier et rustique de nos paysans de France ». Sans le savoir, l'intendant ne fait que reprendre la remarque de Charlevoix, au dire de qui «la rusticité, soit dans le langage, soit dans les façons, n'est même pas connue dans les campagnes les plus écartées ».

Voilà, si l'on y réfléchit un peu, un phénomène surprenant. Il faudrait d'abord s'assurer si Charlevoix et les autres témoins le plus souvent cités ont visité « les campagnes les plus écartées ». Il ne semble pas. Mais on peut se demander si, pour qui désirait entrer en contact avec le gros de la population, il était indispensable de faire de longues expéditions. Il est vrai que les trois quarts des Canadiens habitaient les campagnes. C'est une donnée que l'on nous signale lorsqu'on veut insister sur l'importance des effectifs ruraux. Toutefois, des calculs que j'ai effectués lorsque j'étais à l'Université de Montréal, il ressort qu'au début du XVIIIe siècle, les deux tiers des habitants du Canada vivaient soit dans les villes, soit dans un rayon de quinze milles autour des villes. Les agglomérations urbaines rassemblaient le peuple autour d'elles. Le peuple maintenait avec elles des rapports constants. L'évêque de Québec le rappelle, en 
1742, aux fidèles de tout le pays: "C'est dans les villes que vous trouvez ce qui manque dans vos campagnes; c'est dans les villes où plusieurs de vos enfants reçoivent une éducation chrétienne; . . c'est dans les villes où les hôpitaux sont ouverts pour vous recevoir dans vos infirmités... » On peut s'expliquer ainsi que les colons apparaissent dégagés de toute « rusticité 》 aux yeux d'observateurs qui, d'ailleurs, ne leur sont pas toujours favorables.

Car les témoignages que l'on porte sur les Canadiens ne sont pas toujours flatteurs. À la fin du XVIIe siècle, Denonville écrit, par exemple: «Ce n'est pas qu'il n'y ait de très bonnes gens [dans la colonie], mais le nombre en est moindre que des autres.» Vers la fin du régime français, Desandrouins dira de nos ancêtres qu'ils n'ont qu'une «apparence de religion». De son côté, Kalm nous les montre «consacrant du temps à la prière et au culte extérieur ». Des jugements et des observations, il s'en trouve, on le voit, pour tous les goûts. Et il n'y a là rien d'inexplicable.

Vers 1750 , la société canadienne est toujours une société catholique. Au moment de sa formation, un siècle plus tôt, elle a bénéficié de l'enseignement et de l'exemple de missionnaires qui se situent à la pointe de la réforme catholique. Puis, la colonisation à buts religieux cède le pas à un développement orienté vers des réalisations économiques. Ce ne sont plus les ordres religieux qui surveillent le choix des immigrants. On entend alors Marie de l'Incarnation déplorer qu'il vienne «beaucoup de canaille» en Nouvelle-France. Le clergé, cependant, reste sévère. Un prêtre du Séminaire de Québec voit dans les concerts des occasions de péché. Des confesseurs hésitent à donner l'absolution à des pénitentes qui affectent des coiffures «immodestes».

On se dit: quelle rigueur! Mais prenons garde d'oublier que ce clergé connaissait mieux que nous ses contemporains. Il savait d'expérience ce que nous ne savons que de façon indirecte. Nous avons appris, par exemple, que le budget de l'État comportait chaque année des prévisions pour l'entretien des enfants 
illégitimes. Nous aurions tort de nous moquer trop vite de l'étroitesse de vue des prêtres de cette époque. Surtout, n'allons pas les accuser de jansénisme. En 1758, Bougainville confie à son frère qu'on ne voit pas un janséniste dans le pays et «qu'on se défie même des gens qui savent ce que c'est ».

Que ressort-il de tous ces faits? Des impressions mêlées. En de telles matières, j'estime que ce sont les plus réalistes. Des assertions catégoriques et sans nuances seraient ici de médiocre psychologie tout autant que de mauvaise histoire.

Nous venons - ai-je besoin de le dire ? - d'emprunter une voie de circulation rapide pour traverser, à la plus vive allure possible, la société canadienne du milieu du XVIIIe siècle. Si je voulais résumer ce voyage à travers une époque, je dirais que cette société était intéressante en ceci qu'elle n'était exceptionnelle ni par ce qu'elle avait ni par ce qui lui manquait. Devant elle, on se trouve en présence d'une collectivité américaine qui est de son temps.

En conclusion, je voudrais formuler une remarque que j'ai faite, il y a plusieurs années, à l'occasion d'un cours public sur la civilisation de la Nouvelle-France. Il semble que l'on veuille parfois enseigner l'histoire du Canada non pas tant pour l'expliquer que pour édifier la jeunesse. L'histoire devient ainsi une forme, tout à fait contestable d'ailleurs, d'hagiographie. Il n'est pas impossible que la jeunesse commence par se laisser édifier. Puis vient un moment où celui qui s'est fait ainsi éduquer ouvre des yeux plus critiques sur ce qu'on lui a raconté. Vient un moment - c'est une partie du prix qu'on paie pour devenir un homme - où il perd sa candeur, son charme et sa simplicité d'enfant. Il voit agir partout, autour de lui et au-dessus de lui, des personnages qui ne sont pas des surhommes; lui-même, s'il a quelque lucidité, il se voit agir autrement qu'en surhomme. Il s'étonne qu'il n'existe aucune commune mesure entre ce qu'on lui a dit des hommes d'autrefois et ce qu'il voit des hommes 
d'aujourd'hui. Ceux-ci sont quand même taillés dans la même étoffe que ceux-là.

Alors, qu'est-il tenté de faire ? Et que fait-il effectivement ? Il opte pour le réel, pour la vie courante. Il choisit la médiocrité, qui seule lui paraît désormais normale. Il ne croit plus ni à l'éloquence qu'il entend ni, bien entendu, à celle qu'il lui arrive de pratiquer pour faire comme les autres. Pour avoir, sans qu'il y ait de sa faute, mal compris l'histoire, il peut aller jusqu'à nier l'histoire. Pensera-t-on que l'histoire et la collectivité y gagnent quelque chose ? Reconnaissons donc que ceux qui ont fait le Canada du XVIIIe siècle n'étaient ni plus grands ni plus petits que nature. Ils ont édifié une société à leur image, une société à hauteur d'homme. C'est déjà très beau.

Tout ce que je souhaite, c'est que nos descendants puissent en dire autant de nous.

GuY Frégault,

Sous-ministre des Affaires culturelles

(Québec). 